\title{
Biomechanical overload in multiple tasks with weekly and annual cycle: practical experiences in the field of cleaning and packaging of fruit.
}

\author{
Daniela Colombini *, E. Occhipinti ** \\ * EPM, Research Unit "Ergonomics of Posture and Movement", Don C. Gnocchi Foundation - Milan-Italy \\ ** EPM, Research Unit "Ergonomics of Posture and Movement", Policlinico Ca' Granda Foundation-Milan- \\ Italy
}

\begin{abstract}
The OCRA method is the reference method chosen in ISO (ISO 11228-3) and CEN (EN 1005-5) standards regarding risk assessment and management of upper limbs repetitive movements and exertions. The method consists of two specific tools (OCRA index and OCRA checklist). In this paper special attention will be devoted to the procedures for the analysis of multiple repetitive tasks.

When computing the OCRA index (checklist score) considering the presence of more than one repetitive task, a "traditional" procedure has been previously proposed. This approach, whose results could be defined as "time weighted average", seems to be appropriate when considering rotations among tasks that are performed almost once every hour. On the contrary, when rotation among repetitive tasks is less frequent the "time weighted average" approach could result into an underestimation of the exposure level. For those scenarios an alternative approach is based on a concept that the most stressful task is the minimum starting point. A peculiar procedure allows to exactly estimate the resulting index within this range of minimum to maximum values. It is possible to apply this approach also for job rotation with weekly or monthly or annual cycle typical of agriculture, supermarket, cleaning sectors. This paper shows criteria and results in two working situation: cleaning (weekly cycle) and packaging of fruit (annual cycle).
\end{abstract}

Keywords: OCRA method, repetitive multiple tasks, job rotation, cleaning, packaging of fruit.

\section{Introduction}

As to the preliminary criteria for studying the Work-related musculoskeletal disorders of upper limbs (UL-WMSDs), it is to be underlined that all together organizational and biomechanical factors (frequency of technical actions, stereotypy of movements, use of force, postures and awkward movements, lack of recovery times, actual duration of exposure to repetitive tasks, additional factors such as vibrations, cold, hits, etc) are to be considered as a major determinant of risk presence.

The European data concerning WMSDs evidence that productive sectors more affected by such diseases (after manufacturing) are construction, fishing and agriculture.

These preliminary epidemiological data concerning these particular sectors (characterized by exposure to several different cycle distribution tasks, be it weekly, monthly or yearly), confirm the need to 
tackle more systematic studies on biomechanical load risk and their specific correlated damages.

\section{Aims}

The traditional risk analysis methods on subjects exposed to several repetitive tasks are generally focused on typically "daily" exposure studies $[2,5]$. In many working sectors however (i.e. in agriculture) exposure may vary in duration and type over a longer period (one week; one month; one year).

This study is aimed at:

- defining a specific procedure tackling the preliminary organization and inherent risk analysis of all tasks (characterized by biomechanical overload);

- setting, on the basis of available models for the analysis of daily exposure, some exposure risk analysis multitask models taking into account the turnover along such longer exposure periods.

- present the results of two applications on the field, in cleaning and packaging of fruits sectors.

To validate such models, the specific final result (overall exposure risk index) should be compared with resulting prevalence of workers affected by UL-WMSD. Those data are achievable through active health surveillance programmes; this predictive characteristic is already present in OCRA method exposure to one or more repetitive tasks with daily turnover [4].

The final aim of this research project by EPM Research Unit is to present a simple and practical tool (through a software) allowing to automatically estimate the exposure risk of turnover multitasks over long periods by simply outlining exposure durations of each task in the identified period.

\section{Methods to evaluate the final exposed index: organizational data, intrinsic indices and the mathematical models}

\subsection{General aspects for assessment of exposure levels to jobs characterized by multi-task turnover}

When dealing with an exposure risk assessment (for upper limb biomechanical overload) to multitask works, it is necessary to go through 3 operating stages:
- facing a preliminary organizational study to establish the kind of turnover: the periodicity of the different tasks (implemented by a worker or by the homogeneous group of workers employed in the same tasks in the considered period) repeated in a period of time, daily or weekly or monthly or yearly.

- defining the intrinsec risk level of each task, using the OCRA checklist. Intrinsic level means ascribing to the task a net duration of 440 minutes/shift with 2 breaks, $8-10$ minutes each, and a lunch break of at least 30 minutes.

- applying specific mathematical models assessing exposure to "multitasks".

\subsection{Cyclical turnover and organizational studies on exposure to yearly rotating multiple tasks.}

While in the industry turnover periodicity is typically daily, in other productive sectors this periodicity is longer: for example in agriculture and in packaging of agriculture products (for example fruits) it is typically yearly. Each month of the year is characterized by different processing, each including different tasks. Priority objective to this organizational study stage is identification of workers "homogeneous group carrying out the same tasks" over the year (which and how many workers are involved and which tasks they carry out).

In a preliminarily report it is necessary to know:

- the name of the different tasks carried-out in the year $(\mathrm{A}, \mathrm{B}, \mathrm{C}, \ldots)$

- the task(s) carried out in each month of the year and the number of hours spent for each tasks in each month of the year (Table 1).

With these data the proportional distribution among tasks over the year can be obtained, being derived for each task as a percentage on the total of hours worked in the year (Table 2). The obtained percentages outline the intrinsic time distribution present among developed tasks.

But to better describe the time distribution of the tasks presented in the year, it is necessary to reassess the proportion by weighting them on "worked time constants". Table 3 shows the reference working duration constants expressed in hours/month (160), days/month (20), days/year (220), months work/year (11), worked hours/year (1760).

Starting with reference to the constants reported in Table 3, we can calculate the weighted proportional distribution among the tasks developed in the year. 
The example reported in Table 4 shows this calculation. The total original worked hours/year (from Table 1) are 1680: considering that the constant used is equal to 1760 hours (Table 3), there is a $0.5 \%$ reduction of working activity. The result is that the percentages reported in the column of "weighted proportional distribution" (respect to the constant) are lower that those of intrinsic proportional distribution (Table 2). If by contrast, the total of worked hours exceeds the constant, the $\%$ reported in the column of calibrated proportional distribution will be higher.

Table 1

Example of duration of different tasks carried out by a homogenous group of workers over one year analytically expressed in hours/month (analytical model)

\begin{tabular}{|c|c|c|c|c|c|c|c|c|c|c|c|c|c|}
\hline \multirow[b]{2}{*}{$\begin{array}{l}\text { TYPE OF WORKING } \\
\text { TASKS }\end{array}$} & \multicolumn{13}{|c|}{ DESCRIPTION OF WORKED HOURS / MONTH FOR EACH TASK } \\
\hline & 奀 & 㲐 & $\sum_{\Sigma}^{\frac{\alpha}{1}}$ & $\frac{\alpha}{\alpha}$ & $\gtreqless$ & 岁 & $\stackrel{\succsim}{ٍ}$ & 강 & 占 & চ্ঠ & 을 & 㟧 & $\begin{array}{l}\text { TOTAL } \\
\text { HOURS }\end{array}$ \\
\hline A & & 80 & 160 & & & & & & & 100 & & & 340.00 \\
\hline $\mathrm{B}$ & & & & 160 & 200 & 80 & & & & & & & 440.00 \\
\hline C & & & & & & & & & 200 & & & & 200.00 \\
\hline $\mathrm{D}$ & & & & & & & & & & & 200 & & 200.00 \\
\hline $\mathrm{E}$ & 160 & 80 & & & & & & & & & & & 240.00 \\
\hline $\mathrm{F}$ & & & & & & 80 & 80 & & & & & & 160.00 \\
\hline G & & & & & & & 100 & & & & & & 100.00 \\
\hline Total hours for month & 160 & 160 & 160 & 160 & 200 & 160 & 180 & 0 & 200 & 100 & 200 & 0 & 1680 \\
\hline
\end{tabular}

Table 2

Example of different tasks carried out by a homogeneous group of workers over one year expressed in \% on the total of hours worked in the year (intrinsic proportional distribution among the tasks developed in the year: worked out from Table 3).

\begin{tabular}{|c|c|c|c|c|c|c|c|c|c|c|c|c|c|}
\hline & \multicolumn{13}{|c|}{ ESTIMATE OF PERCENTAGES STARTING FROM WORKED HOURS / MONTH FOR EACH TASK } \\
\hline & 㞯 & 䭴 & $\sum_{\Sigma}^{\frac{\alpha}{\Sigma}}$ & $\frac{\alpha}{\frac{\alpha}{\alpha}}$ & 交 & 岁 & う & 얼 & 占 & চঠ & ठे & 岂 & $\begin{array}{l}\text { Proportional } \\
\text { distribution of } \\
\text { tasks }\end{array}$ \\
\hline$A$ & & $50 \%$ & $100 \%$ & & & & & & & $100 \%$ & & & $20,2 \%$ \\
\hline B & & & & $100 \%$ & $100 \%$ & $50 \%$ & & & & & & & $26,2 \%$ \\
\hline C & & & & & & & & & $100 \%$ & & & & $11,9 \%$ \\
\hline$D$ & & & & & & & & & & & $100 \%$ & & $11,9 \%$ \\
\hline$E$ & $100 \%$ & $50 \%$ & & & & & & & & & & & $14,3 \%$ \\
\hline $\mathrm{F}$ & & & & & & $50 \%$ & $44 \%$ & & & & & & $9,5 \%$ \\
\hline$G$ & & & & & & & $56 \%$ & & & & & & $6,0 \%$ \\
\hline & $100 \%$ & $100 \%$ & $100 \%$ & $100 \%$ & $100 \%$ & $100 \%$ & $100 \%$ & 0 & $100 \%$ & $100 \%$ & $100 \%$ & 0 & $\begin{array}{c}100 \% \\
\text { (on } 1680 \text { h.) }\end{array}$ \\
\hline
\end{tabular}

Table 3

Duration constants of generic working activity to be used to weight exposure duration.

\begin{tabular}{|l|l|l|l|}
\hline Worked hours/ month constant & 160 & Working month constant & 11 \\
\hline Worked days/ month constant & 20.0 & Working hour/ year constant & 1760 \\
\hline Worked days/ year constant & 220 & & \\
\hline
\end{tabular}

3.3 OCRA checklist for assessment of "intrinsic level" of exposure

The OCRA method [1,3], now adopted by ISO (ISO 12228-3) and CEN (EN1005-5) proposes two 
risk analysis tools: OCRA index and OCRA checklist: this last represents the priority analytical model during the first risk assessment stage in a given working situation (mapping stage).
Applying the appropriate mathematical model at the obtained partial scores, describing each risk factor, it allows to assess the final exposure level split in 4 different risk levels (green, yellow, red, purple), as described in Table 5.

Table 4

Example of calculation of weighted proportional distribution (considering the constant) among the tasks carried out in the year and as compared with intrinsic proportional distribution (worked out from Table 3).

\begin{tabular}{|c|c|c|c|c|c|c|c|c|c|c|c|c|c|c|c|}
\hline $\begin{array}{l}\text { WORKING } \\
\text { TASKS }\end{array}$ & 岕 & 岀 & $\underset{\frac{\alpha}{\alpha}}{\frac{\alpha}{z}}$ & $\frac{\text { 品 }}{\frac{\alpha}{\alpha}}$ & $\stackrel{亠}{\Sigma}$ & 岁 & う & 궁 & 点 & চ্ & 으 & 岃 & $\begin{array}{l}\text { TOT. } \\
\text { WORKED } \\
\text { HOURS }\end{array}$ & $\begin{array}{l}\text { INTRINSIC } \\
\%\end{array}$ & $\begin{array}{l}\text { CONSTANT } \\
\text { WEIGHTED } \\
\%\end{array}$ \\
\hline & & & & & & & & & & & & \multicolumn{2}{|r|}{1680} & $100,0 \%$ & $95,5 \%$ \\
\hline $\mathrm{A}$ & & $50 \%$ & $100 \%$ & & & & & & & $100 \%$ & & & 340 & $20,2 \%$ & $19,3 \%$ \\
\hline$B$ & & & & $100 \%$ & $100 \%$ & $50 \%$ & & & & & & & 440 & $26,2 \%$ & $25,0 \%$ \\
\hline $\mathrm{C}$ & & & & & & & & & $100 \%$ & & & & 200 & $11,9 \%$ & $11,4 \%$ \\
\hline $\mathrm{D}$ & & & & & & & & & & & $100 \%$ & & 200 & $11,9 \%$ & $11,4 \%$ \\
\hline$E$ & $100 \%$ & $50 \%$ & & & & & & & & & & & 240 & $14,3 \%$ & $13,6 \%$ \\
\hline $\mathrm{F}$ & & & & & & $50 \%$ & $44 \%$ & & & & & & 160 & $9,5 \%$ & $9,1 \%$ \\
\hline G & & & & & & & $56 \%$ & $100 \%$ & & & & & 100 & $6,0 \%$ & $5,7 \%$ \\
\hline
\end{tabular}

Table 5

The final score of OCRA checklist matched with OCRA index score and related risk ranges.

\begin{tabular}{|l|l|l|l|}
\hline OCRA checklist score & OCRA index score & Exposure levels & $=$ No risk \\
\hline Up to 7.5 & 2.2 & GREEN & $=$ borderline \\
$7.6-11.0$ & $2.3-3.5$ & YELLOW & $=$ light risk \\
$11.1-14.0$ & $3.6-4.5$ & RED LIGHT & $=$ medium risk \\
$14.1-22.5$ & $4.6-9.0$ & RED MEDIUM & $=$ high risk \\
$\geq 22.6$ & $\geq 9.1$ & RED HIGH & \\
\hline
\end{tabular}

\subsection{OCRA checklist for assessment of "intrinsic level" of exposure}

After tackling the first organizational study stage (identification of developed tasks and turnover times, time proportion in the final cyclic period) it is now necessary to determine other organisational data necessary to calculate the net duration of repetitive task and consequently the final risk index like:

- $\quad$ shift net duration for the most representative working shift in a year;

- breaks distribution and duration;

- duration of non repetitive tasks present in a representative shift;

When speaking of intrinsic risk value, we mean assessing each task as if it was the only task developed along the whole shift (approx. 440 net minutes of repetitive task duration with a lunch break of at least 30 minutes and two breaks of minimum 8 minutes).
The second step is to apply, to the intrinsic risk index values, the appropriate OCRA multipliers for specifically modifying them considering both the breaks distribution and the net duration of repetitive task.

3.5 OCRA checklist: assumptions of calculation models for yearly rotation multitask exposure.

Starting from the data derived from the organizational data collections, 2 calculation models were assumed to calculate the multitask annual exposition: a) the traditional weighted average but redefined on time constants; b) complex multitask formula for OCRA checklist (or index).

a) Calculation of "average index weighted by exposure time constant"

It is the same when repetitive task rotation occurs at least every hour during a shift: equation (1). 


\section{Checklist OCRA Multitask Simple = \\ $[(p A \times \% t A)+(p B \times \% t B)+\ldots .+(i . . p N \times \% t N)] \times M d$}

(1)

Where:

"pA", "pB", etc. are the checklist intrinsic scores of each task and $\% \mathrm{tA}, \% \mathrm{tB}$ etc. represent the corresponding duration proportions (in \%) in relation to the constants (Table 3)
$\mathrm{Md}=$ duration multiplier considering the total net duration of repetitive tasks in the shift that is the sum of each repetitive task duration (Table 6)

Calculation of the OCRA checklist final score concerning the workplace investigated with relation to repetitive work net duration.

60-120 $\mathrm{min}$ : Multiplying factor $=0.5$

$121-180 \mathrm{~min}:$ Multiplying factor $=0,65$

$81-240 \mathrm{~min}$ : Multiplying factor $=0.75$

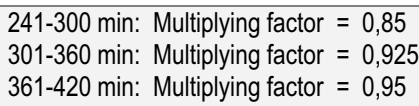

421-480 min: Multiplying factor $=1$

$>480 \mathrm{~min}$ : Multiplying factor $=1,5$
Before calculating this weighted average risk index, it will be necessary to evaluate:

- duration and distribution of breaks and non repetitive tasks

- the net duration of repetitive tasks in a typical day of the year

- the OCRA checklist intrinsic values of each task, re-evaluated considering the actual, above reported, organizational factors present in a typical shift (break distribution and .net duration of repetitive tasks).

b) Calculation of exposure index with the $O C R A$ Multitask Complex model

The calculation uses the application of Multitask Complex Model, the same assumed for daily exposure to several tasks with rotation ( in a daily shift) exceeding one hour: see equation (2).

To be able to calculate Dum $_{\mathrm{i}}$ (duration multiplier of each task in the year), it was devised to:

- reduce the exposure period to a fictitious daily working shift

- transform the yearly exposure proportion (those re-weighted considering duration constant) into fictitious daily shift minutes .

In this case the mathematical model uses the "worst working situation" (the task most at risk recalculated in relation with its real duration as well as with the total duration of all repetitive tasks in the shift). This first estimation is to be weighted with the values and durations of all the other repetitive tasks present in the shift. The complex formula used is the following:

\section{Checklist OCRA Multitask Complex =} score $_{1(\text { Dum } 1)}+\left(\Delta\right.$ score $\left._{1} \times \mathrm{K}\right)$

Where:

$1,2,3, \ldots, \mathbf{N}=$ repetitive tasks ordered by exposure level $(1=$ the highest) using for calculating risk index the Duration multiplier $\left(\right.$ Dum $\left._{i}\right)$ related to their actual duration in the shift.

Dum $_{\mathbf{i}}=$ Duration multiplier considering the actual task duration in the shift

Dum $_{\text {tot }}=$ Duration multiplier considering the total duration of all repetitive tasks in the shift

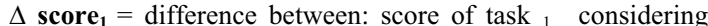
Dum $_{\text {tot }}$ and Score of task $\mathrm{k}_{1}$ considering Dum

$\mathbf{K}=\left(\right.$ score $\left._{1 \max } * \mathrm{FT}_{1}\right)+\left(\operatorname{score}_{2 \max } * \mathrm{FT}_{2}\right)+\ldots+\left(\right.$ score $_{\underline{N}} *$ $\left.\mathrm{FT}_{\mathrm{N}}\right) /\left(\right.$ score $\left._{1 \text { max }}\right)$

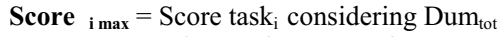

$\mathrm{FT}_{\mathrm{i}}=$ task time fraction (value 0 to 1 ) in relation to the total duration of repetitive tasks.

3.6 Cyclical turnover, organizational studies on exposure to weekly periodicity multitasks and mathematical models for the final risk evaluation

Some working situations clearly show a weekly rotation pattern of repetitive tasks: let us mention the tasks carried out in the kitchens (specially for food preparation in business or school canteens), for some organizational models of cleaning, supermarkets, etc.

In some cases not only tasks vary day by day but also shift duration itself changes over the week.

Just to allow assessment of exposure level, an analytical model was set up essentially recalling the previous one but allowing a more accurate daily diversification. The structure, criteria, information operative steps, needed to obtain the final exposition indices, are the same exposed for multitask exposition (above exposed) with an annual cycle. 
The difference consists that the duration units for the task are now the minutes /day/duration of each task that have be estimated for day of the week.

\section{Results in packaging of fruits (annual cycle)}

The section reports the results of a assessment in an homogeneous group of workers involved in the sorting and packaging the fruit. This group works, every month of the year, a different hours, which increase progressively from January to July, then declined until December: in the summer months it is working peaches (Figure 1).

Different types of fruit are processed that are distributed differently in different months (Figure 2). They're far identified 180 different tasks on which all workers, belonging to the homogeneous group, rotate.

Figure 3 describes briefly the work, highlighting the major risk factors to biomechanical overload.

Given the amount of tasks on which workers rotate, the company was not able to provide exposition times each month for each work task, information necessary to derive the percentage exposition/ year for each task and therefore the risk index final.

The company, however, is aware of how many workstations on the active lines per month for each task (a task can be performed on one or more locations). Since each worker rotates on all these tasks, from these data, it was possible to obtain the $\%$ display for each task, representing the probability of turn on that station. From here we derive the exposition.

We have created a software that can now quickly calculate the final checklist OCRA index. The inputs needed to achieve the final result, as already explained above, are:

- The list of tasks (180!)

- The hours worked per month;
- The description of a representative day of the year for the calculation of the net time of repetitive work (380 minutes in this example) and the duration and distribution of breaks (here are 2 breaks of 10 minutes);

- The calculation of all indices with the intrinsic OCRA checklist index

Obviously the collection of this data (we also hold all the movies of tasks) took a long time but it was observed that in many other companies these tasks are performed in the same way.

The software, built for this specific purpose, makes possible not only within the same company, but also in others industry, to calculate the of the final annual index risk, simply by updating the organizational data without the need to reassess all the of inherent risk indices of each task.

In Figure 4 we show the results of the evaluation indices of an annual exposition. For every month we evaluated the exposition index obtained with the weighted averages calculation mathematical model, The two mathematical models described before, are applied to the calculation the exposition over the year: the weighted average and Multitask Complex where the differences are very evident!

The model shows the highest values for the Multitask Complex. In this peculiar work situation, in which very different durations of work in different months of the year are present, it follows that the exposition is monthly different, going to low risk in winter even at very high risk in the summer months. Using the mathematical model, which uses a weighted average, it will tend to "mediate" the low values with high; otherwise the model Multitask Complex tends to keep the worst values. What will be the best model? They visited the 117 employees: $27 \%$ had at least one UL-WMSDs disease (shoulder tendonitis and CTS)! The model Multitask Complex seems to be in this case the most reliable.

Figure 1

Hours worked (per month) by each worker in the sorting and packaging of fruit.

\begin{tabular}{|c|c|c|c|c|c|c|c|c|c|c|}
\hline \begin{tabular}{l|l}
$\mathbf{1 1 7 4 , 7 1}$ & TOT.NO. OF HOURS/YEAR \\
\end{tabular} & GEN & FEBR & MAR & APR & MAY & JUN & JUL & AGO & SEPT & OCT \\
\hline NO.OF WORKED HOURS/MONTH & 67 & 67 & 105 & 96 & 123 & 147 & 181 & 148 & 81 & 65 \\
\hline $\begin{array}{l}\text { \% CONSIDERING CONSTANT OF } 160 \\
\text { HOURS/MONTH }\end{array}$ & $42 \%$ & $42 \%$ & $66 \%$ & $60 \%$ & $77 \%$ & $92 \%$ & $113 \%$ & $92 \%$ & $51 \%$ & $41 \%$ \\
\hline
\end{tabular}


Figure 2

Different kind of fruits processed during the year

\begin{tabular}{|l|l|l|l|l|l|l|l|l|l|l|l|l|}
\hline FRUITS & GEN & FEBR & MAR & APR & MAY & JUN & JULY & AUG & SEP & OCT & NOV & DIC \\
\hline APPLES & & & & & & & & & & & & \\
\hline KAKY & & & & & & & & & & & & \\
\hline KIWI & & & & & & & & & & & & \\
\hline STRAWBERRIES & & & & & & & & & & & & \\
\hline PLUMS & & & & & & & & & & & & \\
\hline PEACHES & & & & & & & & & & & & \\
\hline APRICOTS & & & & & & & & & & & & \\
\hline
\end{tabular}

Figure 3

Brief decscription of the repetitive task (sorting and packaging)

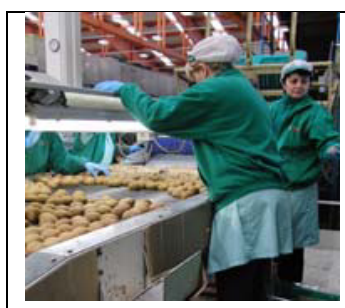

The fruit is selected, discarding the bad. It is then packed in different packages: in trays of various sizes, in bags, in baskets of various sizes. Often the structure of the machinery required to work with arms raised. The hands work in a pinch grip; the frequency of actions is very high and often exceeds the 60 actions per minute. Frequently the large extension of the elbow flexion. There is no strength. The intrinsic values of the OCRA indices, are, for a good part of the tasks performed, in the red area (high or medium risk) or purple (high risk). The rhythms are imposed by the machine: the workers operate on lines scroll continuously.

Figure 4

Synthesis of the assessment results: OCRA scores referred to one year

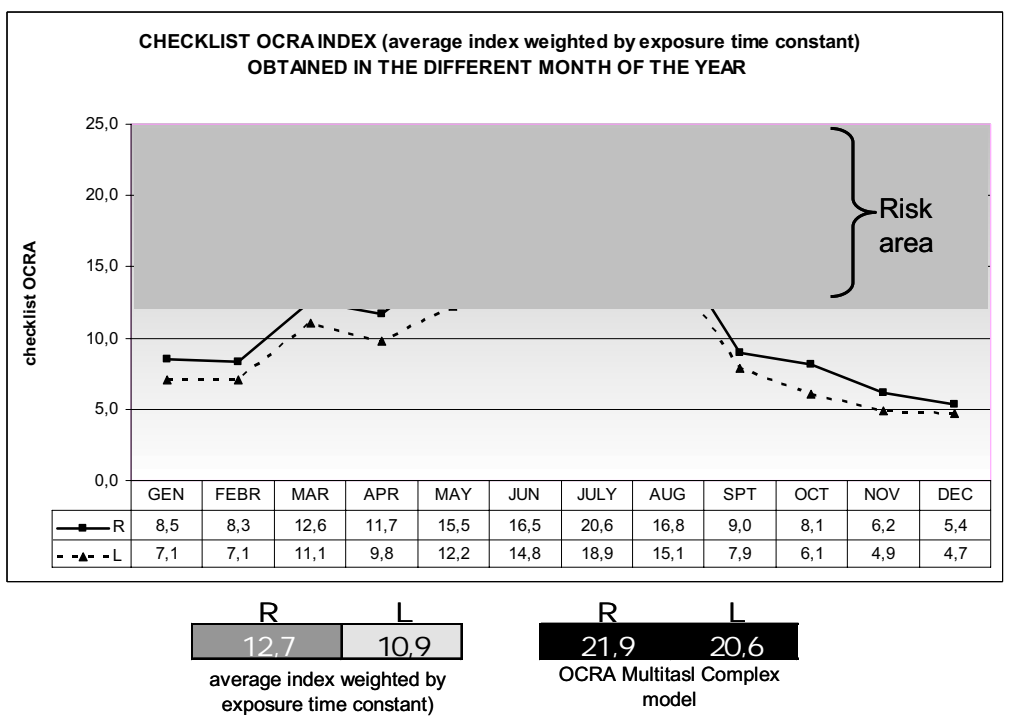

\section{Results in cleaning (weekly cycle).}

Even in the cleaning sector exposure is variable. The cycle is prevalently a week, with the addition of periodic cleaning tasks, performed with frequency that could be very different and may be included in the weekly cycle. The duration and distribution of shifts, with different days of the week involved, is 
highly variable (Figure 5) as well as the tasks assigned. We have identified at least 160 different cleaning tasks. To calculate the exposure a software specifically built was used. For reasons of space it is not possible to present it in detail.

It is important to underline that the calculation and mathematical models are the same as presented for the annual display of multi-tasking.

The calculation of the net exposition time to cleaning operation was based not on the shift time but on: a) the technical content of the cleaning contract $\left(\mathrm{m}^{2}\right.$ open plan, stairs $\mathrm{m}^{2}$, number of rooms, number of bathrooms, number of showers etc. ...); b) predetermined average time for "cleaning unit" (average seconds to clean a shower or wash the floor of an average room of $30 \mathrm{~m}^{2}$,etc .); c) cleaning tasks assignments and their proportional distribution (100\% daily or other percentages depending on the occasion of the event: 2 times a week, once a month, etc. ..).

The average distribution of shifts in the different area of country has been studied in a large cleaning Italian company (3800 workers) The results show that $64 \%$ of workers are engaged 20 hours a week that seem to be, in the most of the cases, in green risk area (absence of risk), rarely in yellow area. For shifts greater than 20-22 hours per week, it is not possible to assume the risk exposition level because it is strongly influenced by both the net times of repetitive work and by the technical content of the cleaning contract. The results of the risk evaluation of a representative sample of 30 homogeneous groups of workers shows that, combining a minimum of 20 hours per week and at least $1200 \mathrm{~m}^{2}$ to clean, it is possible to assume the presence of a risk in yellow area that need health surveillance

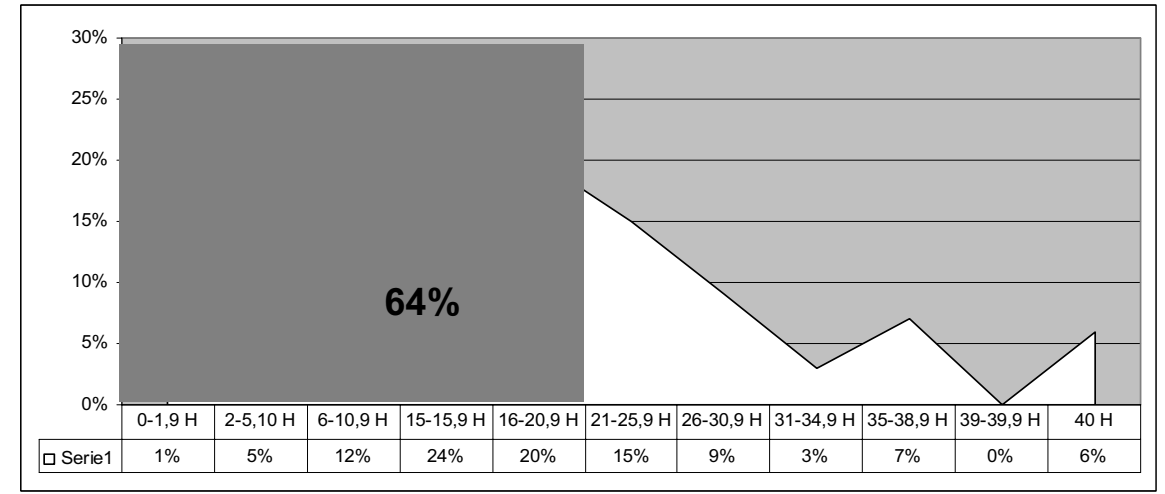

\section{Conclusions}

In some productive areas (agriculture, construction, cleaning, food, etc.) the need is emerging to face upper limb biomechanical overload. Exposure assessment is much more complex being characterised by the presence of several working tasks over periods longer than the current working day (weekly, monthly, yearly turnover).The present work reports organizational study procedures as well as exposure models (starting from OCRA checklist intrinsic values per each identified task) to get to assess the final exposure value via 2 calculation model assumptions..

The future work allowing selection of the most reliable model (because more predictive), will necessarily go through the collection of epidemiological data. Now collection points of this information are active in Italy (Siena University), in Spain and Chile (UPC Barcelona). The first national data on vineyard pruning and olive harvest in the Siena area (approx 90 workers) and other fruit harvesting in the Romagna area (approx 50 workers), packaging fruits (111 workers) seem to confirm once again better prevision for OCRA Multitask Complex model.

In the meanwhile usable software (for cleaning sectors and packaging of fruits) are available and able to provide, once final organizational data have been input as well as different task intrinsic indices, the automatic computing of exposure levels to these extremely complex work organizational models. 


\section{References}

[1] Colombini D, Occhipinti E, Grieco A, 2002. Risk assessment and management of repetitive movements and exertions of upper limbs, Elsevier Science, Amsterdam.

[2] Colombini D, Occhipinti E, 2008. The OCRA Method (OCRA Index and Checklist). Updates with special focus on multitask analysis. Conference Proceedings. AHFE 2008 Las Vegas - July 2008. Eds W. Karkwoski and G. Salvendy. ISBN 978-1-60643-712-4.
[3] Occhipinti, E, 1998. OCRA, a concise index for the assessment of exposure to repetitive movements of the upper limbs. Ergonomics 41, 9, 1290-1311.

[4] Occhipinti E, Colombini D ,2007. Updating reference values and predictive models of the OCRA method in the risk assessment of work related musculoskeletal disorders of the upper limbs (UL-WMSDs). Ergonomics, 50, 11, 1727-1739.

[5] Occhipinti E , Colombini D, Occhipinti M, 2008. Metodo Ocra : messa a punto di una nuova procedura per l'analisi di compiti multipli con rotazioni infrequenti. MEDLAV (La Medicina del Lavoro), 99, 3, 234-241. 